\title{
Comment on: Visinoni et al. The Role of Liver Fructose-1,6-Bisphosphatase in Regulating Appetite and Adiposity. Diabetes 2012;61:1122-1132
}

\author{
Andrea Masotti
}

$\mathbf{I}$ n the May 2012 issue of Diabetes, Visinoni et al. (1) described the role of liver fructose-1,6-bisphosphatase (FBP-1) in regulating appetite and adiposity and in contributing to body weight regulation. In transgenic mice overexpressing $F B P-1$, they observed a lean body weight phenotype and $\sim 50 \%$ reduction of adiposity that was supported by a reduction of food intake. In keeping with this evidence, appetite-stimulating neuropeptide $Y$ and Agouti-related peptide were found to be significantly suppressed. The authors observed an inversion of the lean body weight phenotype by pharmacologic inhibition of FBP-1, suggesting a crucial role for this enzyme in the presence of excess nutrients/fats. FBP-1 is an enzyme in the gluconeogenesis pathway, converting fructose-1,6-bisphosphate to fructose 6-phosphate in the liver. Interestingly, fructose1,6-bisphosphate is also the substrate for another enzyme, aldolase A (ALDOA), which catalyzes the lysis into glyceraldehyde 3-phosphate and dihydroxyacetone phosphate in a reaction activated in the glycolysis process. The glycolysis/ gluconeogenesis pathway therefore might be implicated in body weight regulation.

Incidentally, with the goal of investigating the mechanisms underlying the pathogenesis of nonalcoholic fatty liver disease and finding novel molecular determinants, my colleagues and I have recently analyzed profiles of microRNAs (miRNAs) expressed in liver of rats that were fed a high-fat diet or a high-fat and high-fructose diet (2). miRNAs are $\sim 22 \mathrm{nt}$ noncoding RNAs that control gene expression by base pairing with 3 '-untranslated regions of their regulated transcripts. We reported that after a 3month hypercaloric diet, rats differentially expressed specific miRNAs, genes, and proteins in a regulated manner compared with controls (rats fed a standard diet). Many molecular determinants have been validated experimentally and found to be involved in carbohydrate metabolism, cell glucose homeostasis, lipids and fatty acid metabolism. Among them, we found the downregulation of miRNA-122 and the contemporaneous overexpression of ALDOA in respect to controls. Moreover, high-fat diet-fed or high-fat and high-fructose diet-fed rats displayed an increased body weight and, if not an obese at least a "fat" phenotype (2). It is tempting to follow the authors' findings that the activation of the gluconeogenesis pathway leads to a lean phenotype, and further speculate that its inhibition (i.e., with FBP-1 inhibitors or activation of the glycolytic pathway through ALDOA overexpression) may lead to the opposite (fat) phenotype.

Thus, to gain information about this intriguing hypothesis and to find some genes related to glycolysis/ gluconeogenesis pathway, I predicted all the putative target genes of those miRNAs that we previously found deregulated (2) using an in-house integrated bioinformatics program (3). Noticeably, none of those miRNAs are able to target the FBP-1 gene. Instead, it was surprising to see that some miRNA-targeted genes belong to the neurotrophin signaling pathway (i.e., neurotrophin 3, NTF3), which can be involved in the production of some appetite-stimulating neuropeptides (4). Therefore, not only does the balance between glycolysis and gluconeogenesis processes deserve further investigation (5), but also miRNA expression coupled with bioinformatics/pathway analysis should be considered to unravel and rationalize the "big biological picture" underlying obesity, diabetes, liver and metabolic diseases, as I recently emphasized (6).

\section{ACKNOWLEDGMENTS}

No potential conflicts of interest relevant to this article were reported.

\section{REFERENCES}

1. Visinoni S, Khalid NF, Joannides CN, et al. The role of liver fructose-1,6bisphosphatase in regulating appetite and adiposity. Diabetes 2012;61:1122-1132

2. Alisi A, Da Sacco L, Bruscalupi G, et al. Mirnome analysis reveals novel molecular determinants in the pathogenesis of diet-induced nonalcoholic fatty liver disease. Lab Invest 2011;91:283-293

3. Masotti A, Alisi A. Integrated bioinformatics analysis of microRNA expression profiles for an in-depth understanding of pathogenic mechanisms in non-alcoholic fatty liver disease. J Gastroenterol Hepatol 2012;27:187-188

4. Williams AG, Hargreaves AC, Gunn-Moore FJ, Tavaré JM. Stimulation of neuropeptide Y gene expression by brain-derived neurotrophic factor requires both the phospholipase Cgamma and Shc binding sites on its receptor, TrkB. Biochem J 1998;333:505-509

5. Kim EJ, Kim E, Kwon EY, Jang HS, Hur CG, Choi MS. Network analysis of hepatic genes responded to high-fat diet in C57BL/6J mice: nutrigenomics data mining from recent research findings. J Med Food 2010;13:743-756

6. Masotti A. Mendelian disorders of membrane trafficking. N Engl J Med 2011; $365: 2038$

\footnotetext{
From the Gene Expression-Microarrays Laboratory, Bambino Gesù Children's Hospital, IRCCS, Rome, Italy.

Corresponding author: Andrea Masotti, andrea.masotti@opbg.net.

DOI: $10.2337 / \mathrm{db} 12-0944$

(C) 2012 by the American Diabetes Association. Readers may use this article as long as the work is properly cited, the use is educational and not for profit, and the work is not altered. See http://creativecommons.org/licenses/by -nc-nd/3.0/ for details.
} 